\title{
(2) OPEN ACCESS \\ Skin biopsy and small fibre neuropathies: facts and thoughts 30 years later
}

\author{
Giuseppe Lauria 이 , ${ }^{1}$ Catharina G Faber, ${ }^{2}$ David R Cornblath ${ }^{3}$
}

\begin{abstract}
- Additional supplemental material is published online only. To view, please visit the journal online (http://dx. doi.org/10.1136/jnnp-2021327742).

${ }^{1}$ Department of Clinical Neurosciences, Fondazione IRCCS Istituto Neurologico "Carlo Besta", Milan, Italy ${ }^{2}$ Department of Medical Biotechnology and Translational Medicine, University of Milan,

Milan, Italy

${ }^{3}$ Neurology, Johns Hopkins University, Baltimore, Maryland, USA
\end{abstract}

\section{Correspondence to}

Dr Giuseppe Lauria, Department of Clinical Neurosciences, Fondazione IRCCS Istituto Neurologico "Carlo Besta", Milan 20133, Italy; Giuseppe. LauriaPinter@istituto-besta.it

Received 5 August 2021 Accepted 9 January 2022

\section{ORIGINS}

Between 1989 and 1990, two independent research groups of anatomists and histologists at the Karolinska Institute of Stockholm, Sweden, showed that antibodies against the cytoplasmic protein gene product 9.5 (PGP9.5) could visualise in bright-field immunohistochemistry the rich cutaneous innervation much better than neuropeptides and other antibodies previously $\mathrm{did}^{12}$ (figure 1 ). Their publications would soon widen the interests of neurology to the human skin.

This novel pathological technique was then used to understand the symptoms in a group of patients who had distal neuropathic limb pain, primarily in the feet, but in whom standard neurological examinations, aside from reduced pinprick sensation, standard nerve conduction studies ${ }^{3}$ and even nerve biopsy findings ${ }^{4}$ were normal. The findings of reduced or absent intraepidermal nerve fibres (IENF), either distally alone or in a length-dependent fashion, strongly suggested that the complaints of distal neuropathic limb pain and nearly normal examinations were due to involvement of the small nerve fibres in the skin, primarily those subserving nociception and thermal sensation. This fitted well with classic neurological education of the fundamental importance of clinical-physiological-pathological correlation in neurological disease.

The new protocol for the quantification of IENF density $^{3}$ overcame the first and more complicated one used in the seminal work of 1959 by Arthur and Shelley ${ }^{5}$ (figure 2) and was then used to provide a measurement at different sites. The technique was initially available only at universities with interest in peripheral neuropathies. These academic centres developed, through international collaborations, normative reference values for the different

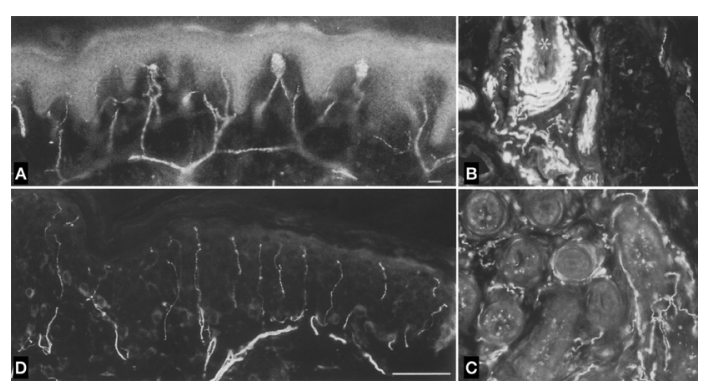

Figure 1 Immunofluorescence micrograph of section of human skin incubated with antibodies to PGP9.1 showing the innervation of the subepidermal area (A), a small artery (B), a sweat gland (C) (adapted from Dalsgaard et al, 1989) and the epidermis from the back (D) (adapted from Wang et al, 1990).

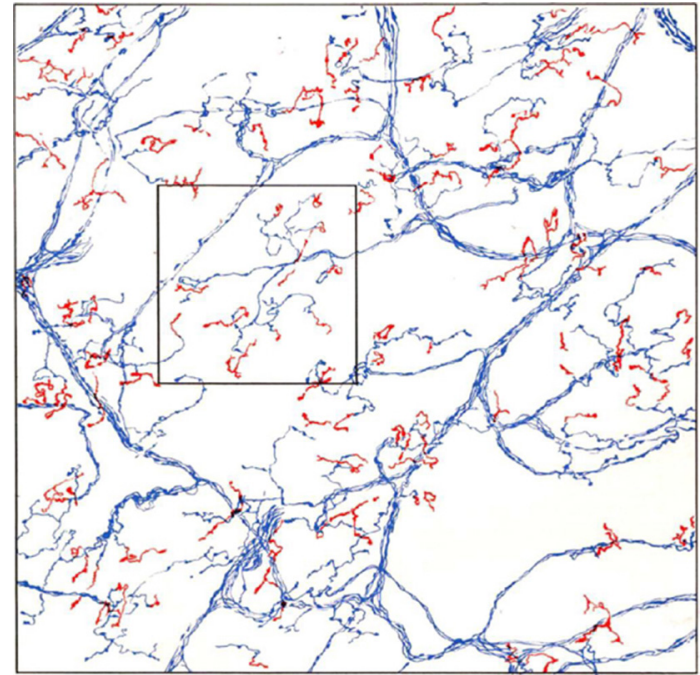

Figure 2 The pattern of cutaneous free-ending innervation. The epidermis in seen from above. Subepidermal nerves are drawn in blue and epidermal nerve in red (adapted from Arthur \& Shilley, 1959).

techniques $^{67}$ and published guidelines, ${ }^{8}$ providing sex and age-adjusted values of normal IENF density at the distal site of the leg for clinical use. This overcame the difficulties related to the development of single centre normative data, which requires the enrolment of very large numbers of healthy individuals. More recently, in the USA at least, commercial laboratories have sprung up claiming to provide the same service. This has led to an explosion of skin biopsies done to evaluate IENF density and a larger explosion of so-called 'small fibre neuropathies' that have gone far beyond the bounds set by classic neurologic clinical-physiological-pathological correlation. How are we to understand these developments?

\section{FUNCTIONAL AND PATHOLOGICAL CORRELATION}

IENF are the endings of small size dorsal root ganglion (DRG) neurons whose expression of the transient receptor potential vanilloid type 1 receptor ${ }^{9}$ reflects their nature as distal nociceptors. IENF lose the enwrapping of non-myelinated Schwann cells while crossing the dermal-epidermal barrier, ${ }^{10}$ like large myelinated sensory nerves do while twisting around dermal mechanoreceptors, ${ }^{11}$ to remain naked among antigen presenting cells (dendritic cells and melanocytes) and keratinocytes which are known to participate in the transduction 
of sensory sensation. ${ }^{12}$ This makes the epidermis, rather than IENF alone, a huge polymodal receptor.

The density of IENF at the distal site of the leg in healthy individuals slightly declines over the decades, using either brightfield or immunofluorescence techniques, and is generally higher in women. ${ }^{67}$ However, the density does not differ between the right and left side, and, most importantly, it does not vary when reassessed after 3 weeks, which is the period of keratinocytes' turnover, both in healthy individuals and in patients with neuropathy, strengthening the diagnostic reliability of the evaluation. ${ }^{13}$

Small size sensory neuron or axon damage can lead either to the loss of thermal and nociceptive sensations, to neuropathic pain, or a combination of both, which is common in several sensory length-dependent neuropathies and non-length dependent neuronopathies. IENF loss seemed able to resolve the clinical-physiological-pathological correlation in almost any process selectively affecting DRG nociceptive neurons or their axons, whenever the related symptoms were appropriate. Indeed, this was reported for patients with congenital insensitivity to pain syndrome, ${ }^{14}$ for those with small fibre neuropathy (SFN) combining neuropathic pain and loss of pinprick sensation, ${ }^{15}$ and for those with painless diabetic neuropathy. ${ }^{16}$ Studies on sensory sensation and neuropathic pain after artificial destruction of these skin nerve fibres, ${ }^{17}$ their spontaneous regrowth in both experimental ${ }^{18}$ and disease-related loss, ${ }^{19}$ and changes in other painful ${ }^{20}$ or painless ${ }^{14}$ diseases confirmed such clinical-physiological-pathological correlation.

Noted early was the lack of correlation between IENF density and neuropathic pain, ${ }^{21} 22$ which in retrospect should not be a surprise. PGP9.5 staining simply detects those skin nerves intact enough to be identified, but it does not tell anything about their functions, which are driven by a precise molecular ontogenesis toward tissue targeting. ${ }^{23}$ Other molecular markers linked to different subtypes of skin nerves might provide a better clinical-physiological-pathological correlation, as suggested by the increased density of IENF expressing peptides in painful diabetic $\mathrm{SFN}^{24}$ and specific molecular signatures for regenerating nerves. ${ }^{25}$

\section{WHAT IS SFN?}

SFN is a disease of somatic, and commonly to a lesser extent autonomic, thin myelinated and unmyelinated nerve fibres. It is called 'pure' when those subtypes of sensory nerves alone are affected, but, more commonly, it occurs as part of a more diffuse impairment of peripheral nerves, of which SFN could be the earliest manifestation.

The classical presentation of SFN is that of a length-dependent neuropathy, a term that links the underlying pathophysiological mechanism of axonal dying-back to the clinical features and anticipates that the feet are first affected by sensory symptoms. Indeed, burning feet has been the eponym used both for familial and sporadic cases. ${ }^{26-28}$ Further clinical presentations have been proposed, from focal neuropathy underlying the cases of burning mouth syndrome ${ }^{20} 29$ or pain after inguinal hernia repair, ${ }^{30}$ in which the distribution of symptoms respected the neuroanatomical distribution, to a non-length dependent presentation reflecting the primary involvement of DRG neurons in paraneoplastic and non-malignant immune-mediated diseases and genetic syndromes. ${ }^{3132}$

The intrinsic limitations of routine nerve conduction studies, psychophysical measurement of thermal thresholds, painrelated evoked potentials and autonomic function evaluation for achieving the diagnosis of SFN in individual patients have been overcome by skin biopsy at the ankle essentially for one reason: the possibility to provide an objective morphometric assessment of the target fibres in the body region corresponding to the symptoms.

Physicians understand that without context an abnormal laboratory value or imaging study has no intrinsic meaning. This perfectly applies also to IENF quantification. Indeed, for neurologists the interpretation of an abnormal IENF density should be just that, nothing more without context. One relevant example is that of sodium channelopathies. Sodium channels are key membrane proteins for the generation and propagation of action potentials and are expressed at low density throughout the length of small nerve fibres. Functional changes caused by gene mutations have been demonstrated in typically painful SFN, but not all patients showed a reduction of IENF. ${ }^{33-35}$ Similarly, patients with inherited erythromelalgia, a severe autosomal dominant syndrome with distal neuropathic limb pain and dysautonomia also caused by sodium channel mutations, can have normal IENF density. ${ }^{36}$ At the other end of the spectrum, patients with congenital insensitivity to pain have profound loss of IENF. ${ }^{37}$ These observations emphasise that epidermal nociceptors can be functionally and structurally altered in disorders causing loss of nociception without pain, and functionally abnormal but structurally normal in some painful syndromes. Similarly, specific sodium channel subunits could be involved in the generation of peculiar neuropathic pain symptoms caused by neurotoxic compounds without evidence of IENF degeneration. ${ }^{38}$

Therefore, the measurement of IENF density at the distal site of the leg is just one among other synergic clues needed to diagnose the classic, length-dependent, SFN. ${ }^{15}$ However, due to widespread use of skin biopsy, the commercial labs that promote it, and interpretative yet deceptive language used in some reports, the diagnosis of 'SFN' is frequently applied to any condition in which IENF at any site is found reduced. This has generated a blurred nosology, because SFN may be virtually (or erroneously) thought of as any disorder with reported loss of IENF, whatever the site of the biopsy, or the distribution or even the type of symptoms.

\section{INTERPRETATION OF RESULTS IN THE CLINICAL CONTEXT}

The loss of IENF is expected to lead to symptoms and clinical signs correlated to the physiological functions of small nerve fibres. Indeed, the old paradigm that SFN patients can complain of severe burning pain and yet have normal clinical examination has been forgotten. In most patients with SFN, the clinical picture is characterised by positive (eg, hyperalgesia) and/or negative (eg, loss of nociception) signs of small fibre dysfunction. ${ }^{15}$ Moreover, when looking at how symptoms correlate with clinical signs and the results of the diagnostic testing, a large study demonstrated that nearly $90 \%$ of patients with neuropathic-sounding symptoms but no clinical signs at examination had normal results for both quantitative sensory testing (QST) and IENF density, which remained normal after 18 months. This suggests that the diagnosis of SFN should not rely on symptoms alone, because they are not sufficiently specific.

Taking SFN as the benchmark, the same correlation should be expected also for other conditions in which there is loss of IENF, whatever is its distribution (eg, length-dependent or independent, focal). Conversely, this does not seem to be always the case.

One example is that of fibromyalgia, a clinical condition diagnosed by the presence of 'multi-site' pain, meaning that six out of nine sites are painful to the patient. ${ }^{39}$ Due to the lack of any clinical and electrophysiological evidence of large fibre sensory 
nerve involvement, fibromyalgia patients have been investigated by skin biopsy and other methods for assessing small fibre functions, with conflicting results. Some have found diffuse IENF loss correlating with the disease severity. ${ }^{40}$ Others reported little contribution from peripheral small nerve fibres as compared with central pain processing impairment [s41], and yet others did not report clinically meaningful correlations between diagnosis of fibromyalgia and small nerve fibre pathway functioning [s42], thus challenging the definition of the small fibre pathology attributed to this disorder [s43].

This lack of correspondence between the distribution of painful symptoms and the site of skin biopsy also hinders the definition of the plausible neuroanatomical correlation required for diagnosing neuropathic pain [s44], contributing to such a blurred scenario. While the loss of IENF assessed in the correspondent clinical area allowed meeting this diagnostic requirement in focal painful syndromes like burning mouth ${ }^{20}$ and notalgia paresthetica [s45], how could one or more skin biopsies at the lower limb address this issue in patients with either nonlocalised pain or pain localised to another site?

For example, some patients with chronic pelvic and bladder pain have been diagnosed with SFN based on one skin biopsy taken at the lower extremity [s46, s47]. However, when vulvar tissue was examined the density of IENF was found to be higher than in healthy controls, indicating that if an alteration of IENF did exist, it was not represented by loss of nerve fibres [s48].

More importantly, how are we to interpret skin biopsy findings in patients without appropriate clinical signs to suggest loss of the distal nociceptive and thermal fibres? The literature is increasingly filled with reports of SFN in several conditions where the a priori view would be that these distal small fibres are not involved [s49]. These include various conditions of central dysautonomia like postural orthostatic tachycardia syndrome [s50], Ehlers-Danlos syndrome [s51] or after vaccination [s52].

Recently, autism joined this list. Autism is a complex neurodevelopmental disorder in which a variety of molecular mechanisms likely underpin the clinical phenotype, which includes hypersensory and hyposensory responsiveness, and sensationseeking [s53]. A recent paper reported the reduction of IENF at the distal site of the leg in about half of 32 adult autistic patients [s54]. Leaving aside the changes in psychophysical somatosensory thresholds and of contact heat evoked potentials amplitude, which might not be surprising within the complex multisystem sensory processing impairment of autism [s55], the simple association between reduced IENF density at one leg and tactile and autistic symptoms seems quite a large conceptual jump.

In some clinical situations, neurologists might expect a loss of IEFN, those being mainly complex neurodegenerative diseases. While many of these affects one neuronal population primarily, affectation to a minor degree of other neuronal populations might not be unexpected. Long ago, Professor P J Dyck showed that some patients with amyotrophic lateral sclerosis had large fibre neuropathy [s56], so it is not surprising that some also have reduction in IENF as indeed it was found [s57], and that insights on possible molecular mechanisms were described [s58]. The same logic would extend to Parkinson's disease, in which neuropathy possibly attributed to L-dopa therapy has been first reported in few patients [s59], then found to involve unmyelinated axons from autopsy sural nerve biopsies [s60], and eventually confirmed by skin biopsies [s61], even though no underlying pathophysiological mechanisms has been proposed yet. It is unclear whether this could apply to other disorders based on one single anecdotal observation like fragile-X syndrome [s62] or when the diagnosis is made on screening questionnaires like for Pompe disease [s63].

\section{IMPLICATIONS ON TREATMENT}

The popularity of the idea that small nerve fibres could be affected in any disease, even lacking any clinical-physiological correlation, and being at the same time easily diagnosed, brings a number of concerns. Among these, the most important for many reasons is proposing disease-modifying treatments with unknown corresponding pathophysiological mechanisms nor evidence of efficacy from properly designed clinical trials. Intravenous immunoglobulin (IVIg) is in the top five drug categories in terms of annual spending in the USA and the yearly in-hospital cost per patient is about US\$60 000 [s64]. In recent years, the hypothesis that patients with SFN associated with systemic immune-mediated diseases could benefit from IVIg originated from retrospective, anecdotal or small open-label studies [s6567]. Following them, and with the same uncontrolled approach, this hypothesis of IVIg efficacy spread from fibromyalgia[68] to 'apparently' autoimmune SFN of adults and children [s69, s70]. However, the largest of these studies showed a 1-point improvement of pain relief in a $0-10$ scale [s70], which is hardly clinically meaningful as this is the same magnitude of change in the placebo group in placebo-controlled studies of pain [s71]. One answer arrived from the first randomised controlled trial designed to investigate if IVIg had any efficacy in providing SFN patients with pain relief. The administration of IVIg ( $2 \mathrm{~g} / \mathrm{kg}$ body weight) or placebo, followed by infusions of IVIg $(1 \mathrm{~g} / \mathrm{kg})$ or placebo at 3 -week intervals in 60 skin biopsy-proven idiopathic SFN did not change the Pain Intensity Numerical Rating Scale score at 12 weeks compared with baseline [s72]. Even though the result of this trial cannot be extended to any disorder classified as SFN, it suggests that controlled trials are mandatory before suggesting the effectiveness of IVIg or any other treatment.

\section{WHAT ARE THE LESSONS?}

Reduced IENF in skin biopsy, like many tests, is a finding, not a diagnosis, which in medicine requires at least two clues converging within the clinical context. SFN should not be regarded as an exception.

IENF density results need to be compared with a control population usually using published data, which are available for the two most commonly used techniques. Developing local controls without an interobserver quality control programme or controls for a specific condition is fraught with potential problems especially if a small sample size is used. The site where biopsy is taken is also important as control data do not exist for many sites now being biopsied such as the top of the foot. Moreover, data should be shown as both mean with SD/SE, mean with range/IQR, but also as percentage of truly abnormal by a predefined outcome measure. Some laboratories report values as 'low normal'. Currently, this has no meaning and certainly no diagnostic significance.

In clinical studies either focusing on diagnosis or efficacy of treatments, blinding of skin biopsy reading is critical and should be specified in the protocol.

Adding skin biopsy to diagnostic criteria for a disease should be carefully considered. Rather than jumping to conclusions, understanding how the biopsy results might fit into the clinical context should be pursued. Some have used the biopsy alone, divorced from the clinical context, to diagnose SFN and to obtain authorisation for IVIg or other putative treatments. We have grave reservations about this line of thinking and see no 
justification for IVIg in SFN aside from within well-designed controlled clinical trials, as recently demonstrated [s72].

While skin biopsy for IEFN density provides a pathological view of the state of small nociceptive and thermal fibres, the simple reduction of IENF, whatever its degree, when blind to the clinical context makes it impossible to predict symptoms, signs and aetiology of the underlying neuropathic process that could affect DRG nociceptors or their axons. Without denying the skin biopsy findings in some patients dissociated from the clinical picture, the hypothesis that assessing IENF at one lower limb may explain complex sensory symptoms or those localised in different body areas seems misguided. This is not trivial, as it has to do with the nosology of a disorder and the consequent diagnostic criteria applied in clinical practice and trials.

Thirty years after the appearance of skin biopsy, which has widened the diagnostic toolbox of neurologists providing structural information on nerves used to be considered clinically invisible, a new step toward the interpretation of the results should be undertaken to make it a measure for the improvement of the diagnostic reliability in the disorders potentially affecting small nerve fibres.

\section{Contributors GL has drafted the work to which all the authors have equally contributed.}

Funding The authors have not declared a specific grant for this research from any funding agency in the public, commercial or not-for-profit sectors.

Competing interests David Cornblath is an Editorial Board Member at Journal of Neurology, Neurosurgery and Psychiatry.

\section{Patient consent for publication Not applicable.}

Ethics approval This study does not involve human participants.

Provenance and peer review Not commissioned; externally peer reviewed.

Supplemental material This content has been supplied by the author(s). It has not been vetted by BMJ Publishing Group Limited (BMJ) and may not have been peer-reviewed. Any opinions or recommendations discussed are solely those of the author(s) and are not endorsed by BMJ. BMJ disclaims all liability and responsibility arising from any reliance placed on the content. Where the content includes any translated material, BMJ does not warrant the accuracy and reliability of the translations (including but not limited to local regulations, clinical guidelines, terminology, drug names and drug dosages), and is not responsible for any error and/or omissions arising from translation and adaptation or otherwise.

Open access This is an open access article distributed in accordance with the Creative Commons Attribution Non Commercial (CC BY-NC 4.0) license, which permits others to distribute, remix, adapt, build upon this work non-commercially, and license their derivative works on different terms, provided the original work is properly cited, appropriate credit is given, any changes made indicated, and the use is non-commercial. See: http://creativecommons.org/licenses/by-nc/4.0/.

\section{ORCID iD}

Giuseppe Lauria http://orcid.org/0000-0001-9773-020X

\section{REFERENCES}

1 Dalsgaard CJ, Rydh M, Haegerstrand A. Cutaneous innervation in man visualized with protein gene product 9.5 (Pgp 9.5) antibodies. Histochemistry 1989;92:385-90.

2 Wang L, Hilliges M, Jernberg T, et al. Protein gene product 9.5-immunoreactive nerve fibres and cells in human skin. Cell Tissue Res 1990;261:25-33.

3 McCarthy BG, Hsieh ST, Stocks A, et al. Cutaneous innervation in sensory neuropathies: evaluation by skin biopsy. Neurology 1995;45:1848-55.

4 Holland NR, Crawford TO, Hauer P, et al. Small-Fiber sensory neuropathies: clinical course and neuropathology of idiopathic cases. Ann Neurol 1998;44:47-59.

5 Arthur RP, Shelley WB. The innervation of human epidermis. J Invest Dermatol 1959;32:397-411.

6 Lauria G, Bakkers M, Schmitz C, et al. Intraepidermal nerve fiber density at the distal leg: a worldwide normative reference study. J Peripher Nerv Syst 2010;15:202-7.

7 Provitera V, Gibbons $\mathrm{CH}$, Wendelschafer-Crabb G, et al. A multi-center, multinational age- and gender-adjusted normative dataset for immunofluorescent intraepidermal nerve fiber density at the distal leg. Eur J Neurol 2016;23:333-8.

8 Lauria G, Hsieh ST, Johansson 0. European Federation of neurological Societies/ Peripheral nerve Society guideline on the use of skin biopsy in the diagnosis of small fiber neuropathy. J Periph Nerv Syst 2010;15:79-92.
9 Lauria G, Morbin M, Lombardi R, et al. Expression of capsaicin receptor immunoreactivity in human peripheral nervous system and in painful neuropathies. $J$ Peripher Nerv Syst 2006;11:262-71.

10 Lauria G, Borgna M, Morbin M, et al. Tubule and neurofilament immunoreactivity in human hairy skin: markers for intraepidermal nerve fibers. Muscle Nerve 2004:30:310-6.

11 Nolano M, Provitera V, Crisci C, et al. Quantification of myelinated endings and mechanoreceptors in human digital skin. Ann Neurol 2003;54:197-205.

12 Lumpkin EA, Caterina MJ. Mechanisms of sensory transduction in the skin. Nature 2007:445:858-65.

13 Lauria G, Dacci P, Lombardi R, et al. Side and time variability of intraepidermal nerve fiber density. Neurology 2015;84:2368-71.

14 Nolano M, Crisci C, Santoro L, et al. Absent innervation of skin and sweat glands in congenital insensitivity to pain with anhidrosis. Clin Neurophysiol 2000;111:1596-601.

15 Devigili G, Rinaldo S, Lombardi R, et al. Diagnostic criteria for small fibre neuropathy in clinical practice and research. Brain 2019;142:3728-36.

16 Bönhof GJ, Strom A, Püttgen S, et al. Patterns of cutaneous nerve fibre loss and regeneration in type 2 diabetes with painful and painless polyneuropathy. Diabetologia 2017:60:2495-503.

17 Simone DA, Nolano M, Johnson T, et al. Intradermal injection of capsaicin in humans produces degeneration and subsequent reinnervation of epidermal nerve fibers: correlation with sensory function. J Neurosci 1998;18:8947-59.

18 Polydefkis M, Hauer P, Sheth $\mathrm{S}$, et al. The time course of epidermal nerve fibre regeneration: studies in normal controls and in people with diabetes, with and without neuropathy. Brain 2004;127:1606-15.

19 Lauria G, McArthur JC, Hauer PE, et al. Neuropathological alterations in diabetic truncal neuropathy: evaluation by skin biopsy. J Neurol Neurosurg Psychiatry 1998;65:762-6.

20 Lauria G, Majorana A, Borgna M, et al. Trigeminal small-fiber sensory neuropathy causes burning mouth syndrome. Pain 2005;115:332-7.

21 Shun C-T, Chang Y-C, Wu H-P, et al. Skin denervation in type 2 diabetes: correlations with diabetic duration and functional impairments. Brain 2004;127:1593-605

22 Devigili G, Tugnoli V, Penza P, et al. The diagnostic criteria for small fibre neuropathy: from symptoms to neuropathology. Brain 2008;131:1912-25.

23 Bartesaghi L, Wang Y, Fontanet P, et al. PRDM12 is required for initiation of the nociceptive neuron lineage during neurogenesis. Cell Rep 2019;26:3484-92.

24 Karlsson P, Provitera V, Caporaso G, et al. Increased peptidergic fibers as a potential cutaneous marker of pain in diabetic small fiber neuropathy. Pain 2021;162:778-86.

25 Baskozos G, Sandy-Hindmarch O, Clark AJ, et al. Molecular and cellular correlates of human nerve regeneration: ADCYAP1/PACAP enhance nerve outgrowth. Brain 2020;143:2009-26.

26 Chan AC, Wilder-Smith E. Clinical Reasoning: burning hands and feet. Neurology 2015;84:e146-52.

27 Kuhlenbaumer G, Young P, Kiefer R, et al. A second family with autosomal dominant burning feet syndrome. Ann N Y Acad Sci 1999;883:445-8.

28 Stögbauer F, Young P, Kuhlenbäumer G, et al. Autosomal dominant burning feet syndrome. J Neurol Neurosurg Psychiatry 1999;67:78-81.

29 Penza P, Majorana A, Lombardi R, et al. "Burning tongue" and "burning tip": the diagnostic challenge of the burning mouth syndrome. Clin J Pain 2010;26:528-32.

30 Aasvang EK, Brandsborg B, Christensen B, et al. Neurophysiological characterization of postherniotomy pain. Pain 2008;137:173-81.

31 Lauria G, Sghirlanzoni A, Lombardi R, et al. Epidermal nerve fiber density in sensory ganglionopathies: clinical and neurophysiologic correlations. Muscle Nerve 2001;24:1034-9.

32 Provitera V, Gibbons CH, Wendelschafer-Crabb G, et al. The role of skin biopsy in differentiating small-fiber neuropathy from ganglionopathy. Eur I Neurol 2018;25:848-53.

33 Faber CG, Lauria G, Merkies ISJ, et al. Gain-Of-Function Nav1.8 mutations in painful neuropathy. Proc Natl Acad Sci U S A 2012;109:19444-9.

34 Hoeijmakers JGJ, Han C, Merkies ISJ, et al. Small nerve fibres, small hands and small feet: a new syndrome of pain, dysautonomia and acromesomelia in a kindred with a novel Nav1.7 mutation. Brain 2012;135:345-58.

35 Huang J, Han C, Estacion M, et al. Gain-of-function mutations in sodium channel $\mathrm{Na}(\mathrm{v}) 1.9$ in painful neuropathy. Brain 2014;137:1627-42.

36 Bennett DLH, Woods CG. Painful and painless channelopathies. Lancet Neurol 2014:13:587-99.

37 McDermott LA, Weir GA, Themistocleous AC, et al. Defining the Functional Role of $\mathrm{Na}_{v} 1.7$ in Human Nociception. Neuron 2019;101:905-19.

38 Chen L, Huang J, Benson C, et al. Sodium channel Nav1.6 in sensory neurons contributes to vincristine-induced allodynia. Brain 2020;143:2421-36.

39 Arnold LM, Bennett RM, Crofford L, et al. AAPT diagnostic criteria for fibromyalgia. J Pain 2019;20:611-28.

40 Evdokimov D, Frank J, Klitsch A, et al. Reduction of skin innervation is associated with a severe fibromyalgia phenotype. Ann Neurol 2019;86:504-16. 